\title{
Effects of stimulus comparison during discrimination training on subsequent transposition and generalization gradients'
}

LEWIS B. SACHS, West Virginia University, Morgantown, W. Va. 26506

Only pigeons in a comparison group were afforded opportunity for direct comparison between stimuli on the same dimension. Following discrimination learning both a comparison and a noncomparison group were given postdiscrimination generalization tests and transposition tests. The results provided further evidence for a correspondence between postdiscrimination and transposition data. However, this correspondence was not complete, as Ss without peak shifts produced statistically significant transposition scores. Also the comparison group produced a significantly greater amount of transposition. Differences in transposition scores between the comparison and noncomparison groups, however, were not reflected in underlying differences in the postdiscrimination generalization gradients.

The explanation of transposition has long been controversial. Varying the opportunity for stimulus comparison during discrimination training has led to divergent predictions and thereby has served as a test of opposing explanations of transposition. According to the "absolute" viewpoint, varying the opportunity for stimulus comparison will not affect generalization gradients and therefore has no effect on the amount of transposition (Spence, 1937). However, according to the "relational" viewpoint, comparison emphasizes the relationship between stimuli and therefore would increase the amount of transposition (Kohler, 1929).

Baker \& Lawrence (1951) specified some of the methodological problems involved in this research. However, recent researchers (Thompson, 1955; Honig, 1962) have controlled variables that had previously confounded the data. The present study was designed to incorporate these methodological improvements and to provide data on both transposition and generalization after comparison and noncomparison discrimination training. Comparison training allowed the stimuli on the same dimension to appear together. Noncomparison training did not allow stimuli along the same dimension to appear together.

The major purpose of this study was to investigate further the effect of altering the opportunity for stimulus comparison on subsequent transposition and postdiscrimination generalization gradients; and primarily, to determine if differences in the amount of transposition varied as a function of differences in underlying postdiscrimination generalization gradients.

\section{SUBJECTS}

Six naive white Carneaux pigeons, maintained at $75 \%$ free-feeding weight, served as Ss.

\section{APPARATUS AND PROCEDURE}

The apparatus was a standard Grason-Stadler pigeon chamber with a digital display unit, mounted behind two keys, which could project 12 different stimulus values. The stimulus values were nine angles $(15,30,45$, $60,75,90,105,120$, and $135 \mathrm{deg})$ and three circles $(1 / 4,1 / 2$, and $3 / 4$ in. in diam).

Following magazine training, Ss received 15 days of single-stimulus training with generalization tests occurring after 5,10 , and 15 days of training. Each session of single-stimulus training consisted of a $60-\mathrm{sec}$ variable interval schedule in which responding to the 75-deg angle was reinforced by a 3-sec presentation of grain. Each session had 9030 -sec stimulus-on periods, each separated by 10 -sec stimulus-off periods. During generalization tests the nine angles were presented for 30-sec periods separated by 10 -sec stimulus-off periods. All generalization tests were carried out under extinction.

The comparison group (Birds 2, 4, and 6) received discrimination training on the two pairs of stimuli in a manner which afforded them direct comparison between stimuli on the same dimension. For the angle pair, the 75-deg angle was positive and the 90-deg angle was negative. For the circle pair, the $1 / 2$-in. circle was positive and the $3 / 4$-in. circle was negative. The criterion for discrimination was reached during the daily session in which a block of five consecutive angle presentations produced at least 75 responses to the positive stimulus and no more than three responses to the negative
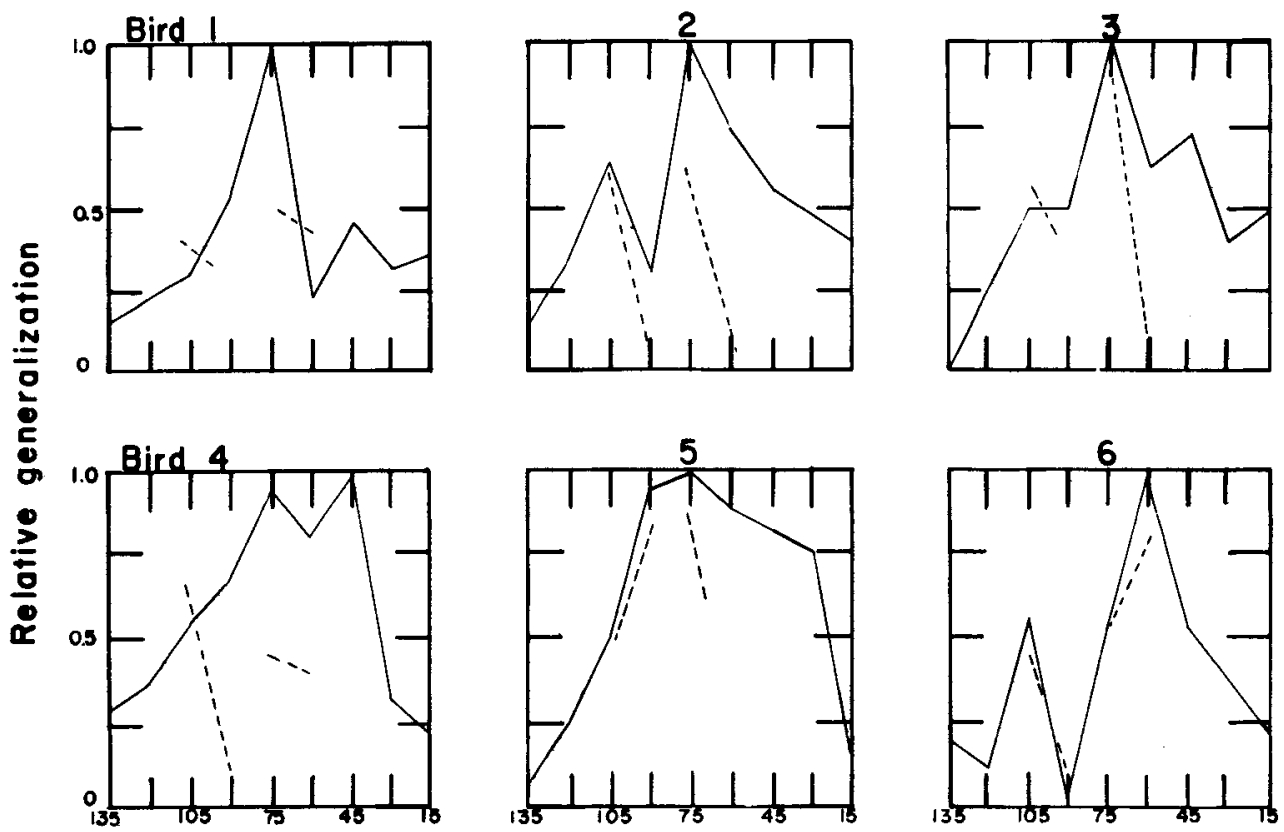

Angle size (degrees)

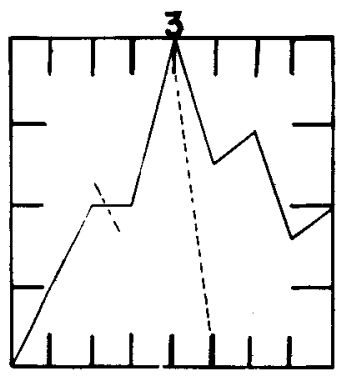

Fig. 1. Individual gradients of the second postdiscrimination generalization test for all Ss. The dashed lines present the results of the two transposition tests that were inserted during the second postdiscrimination generalization tests. 


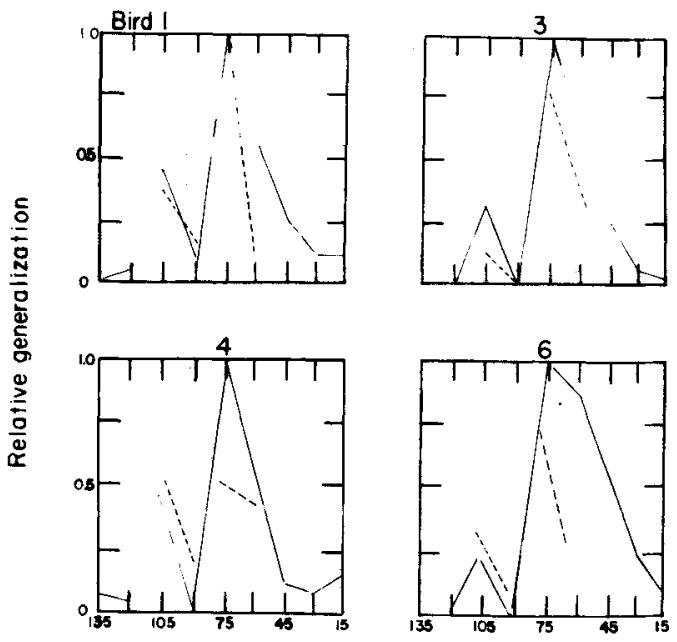

Angle size (degrees)

Fig. 2. Individual gradients of the postdiscrimination generalization tests for the successively trained Birds $1,3,4$, and 6 . The dashed lines present the results of the two transposition tests that were inserted during the postdiscrimination generalization tests.

stimulus. Within the same daily session, it was also required that $\mathrm{Ss}$ attain the same criterion for a block of five consecutive circle presentations.

The noncomparison group (Birds 1,3, and 5) received discrimination training in a manner that did not provide direct comparison. For the first pair, the 75 -deg angle was positive and the $3 / 4$-in. circle was negative. For the second pair, the $1 / 2$-in. circle was positive and the 90 -deg angle was negative. The criterion level for these two discriminations was the same as for the comparison group.

Upon the attainment of criterion for discrimination training, Ss were given transposition tests, consisting of the simultaneous presentation of the 75 -deg angle and the 60-deg angle. Transposition tests were randomly inserted within the daily sessions of discrimination training. Transposition is well is postdiscrimination generalization tests were carried out under extinction.

Subjects were continued on discrimination training un til they once again at tained the original criterion and then given two further postdiscrimination generalization tests along with further transposition tests. The transposition tests now included the simultaneous presentation of the 105-deg angle and the 90-deg angle.

The first four Ss (Birds 1, 3, 4, and 6) to complete the above procedure were given five days of successive discrimination training with the 75-deg angle as positive and the $90-\mathrm{deg}$ angle as negative. This was again followed by transposition tests and postdiscrimination generalization tests.

\section{RESULTS}

The results of the generalization tests indicated that each $S$, on at least the first, second, or third generalization gradient, peaked at the conditioned stimulus, and produced a decreased number of responses on either side. There was no statistically significant differences between the comparison and noncomparison group on the attainment of the discrimination criterion.

There were significantly more responses to the 60-deg angle (transposed stimulus) than to the 75-deg angle on the initial day of transposition tests over all Ss $(t=4.04$, df $=5, p<.01)$. The comparison group produced a significantly greater number of transposition responses over the four days of testing than did the noncomparison group $(\mathrm{t}=3.48, \mathrm{df}=5, \mathrm{p}<.01)$.

The results of the first postdiscrimination generalization tests were very similar to the results of the generalization tests given prior to discrimination training. Only one $S$ displayed a postdiscrimination peak shift. The results of the second postdiscrimination generalization tests given after the Ss once again returned to criterion discrimination and results of both the transposition tests are presented in Fig. 1. The results of transposition were in relative agreement with the results of the postdiscrimination generalization gradients as there were only three exceptions.

The results of the postdiscrimination generalization gradients for the successive discrimination Ss are presented in Fig. 2. The transposition tests were completely congruent with the generalization gradients.

A notable feature of the postdiscrimination generalization gradients was the pattern of elevation of responses to the 105-deg angle demonstrated by several Ss.

The most consistent differentiating factor between the prediscrimination and postdiscrimination gradients was the pattern of an elevation of the number of responses made to the 105-deg angle and the decrement in the number of responses made to the 90-deg angle. Both the 105-deg and the 75-deg angle represented 15-deg angles either to the left or the right side of a vertical frame of reference and both represented 75-deg angles either to the left or right side of a horizontal frame of reference. Therefore, the disproportionately high number of responses at the 105-deg angle might have been a function of stimulus generalization. Mello (1965) has demonstrated that pigeons reinforced for peaking at a 45 -deg oblique line with one eye would respond maximally to the mirror-image, a 135-deg oblique line with the opposite eye. Therefore, interocular reversal might also have constituted a possible explanation for the disproportionately high number of responses to the 105-deg angle.

The inclusion of the 105-deg angle vs the 90-deg angle transposition test was expected to have been a crucial index of the relationship between transposition and postdiscrimination generalization gradients. That is, the unilateral nature of the peak shift would have ruled out a peak-shift explanation for any obtained transposition on the 105-deg angle vs the 90-deg angle pair. For successive discrimination, this index would also have constituted a test of Honig's (1962) results concerning the congruence or lack of congruence between results obtained from a single-stimulus generalization gradient and the results obtained from a double-stimulus generalization gradient. Unfortunately, it was not possible to interpret the data in regard to this index since the pattern of an elevation of the number of responses to the 105-deg angle contaminated any such at tempted evaluation.

Previous research and data from this investigation provide evidence for a close correspondence between postdiscrimination generalization gradients and transposition. However, this correspondence was not complete, as only one $\mathrm{S}$ demonstrated a peak shift in the first postdiscrimination generalization gradient despite the fact that all Ss had just previously indicated a statistically significant amount of transposition. Also the results of this investigation indicated that the opportunity for stimulus comparison produced a significantly greater amount of transposition. The difference in transposition scores between the comparison and noncomparison groups, however, was not reflected in underlying differences in the postdiscrimination generalization gradients.

\section{REFERENCES}

BAKER, R. A., \& LAWRENCE, D. H. The differential effects of simultaneous and successive stimuli presentation on transposition. Journal of Comparative \& Physiological Psychology, 1951, 44, 378-382.

HONIG, W. K. Prediction of preference, transposition, and transpositionreversal from the generalization gradient. Journal of Experimental Psychology, 1962, 64, 239-248.

KÖHLER, W. Gestalt psychology. New York: Liveright, 1929.

MELLO, N. K. Interhemispheric reversal of mirror-image oblique lines after monocular training in pigeons. Science, 1965, 148, 252-254.

SPENCE, $K$. W. The differential response in animals to stimuli varying within a single dimension. Psychological Review, 1937, 44, 430-441.

THOMPSON, $R$. Transposition in the white rat as a function of stimulus comparison. Journal of Experimental Psychology, 1955, 50, 185-190. NOTE

1. This paper was based on a dissertation submitted to the Department of Psychology at Washington State University (1966). Grateful acknowledgement is due Dr. Kenneth E. Lloyd, who provided assistance with the research and this manuscript. 